\title{
THE TRAVELING WAVE OF AUTO-CATALYTIC SYSTEMS - THE LIMITING CASES
}

\author{
YUANWEI QI \\ ${ }^{1}$ Department of Mathematics \\ University of Central Florida \\ Orlando, FL 32828, USA
}

ABSTRACT: This article studies propagating wave of a reaction-diffusion system modeling auto-catalytic chemical reaction $A+n B \rightarrow(n+1) B$ involving two chemical species, a reactant $A$ and an auto-catalyst $B$, whose diffusion coefficients, $D_{A}$ and $D_{B}$, are unequal due to different molecular weights and/or sizes.

We are investigating the extreme cases of $D \equiv D_{B} / D_{A} \ll 1$ or $D \gg 1$.

We reformulate the traveling wave problem of general case to derive explicit bounds of speed for existence and non-existence.

AMS Subject Classification: 34C20, 34C25, 92E20

Received: June 13, 2017; $\quad$ Accepted: October 31, 2018 ;

Published: November 13, 2018. doi: 10.12732/caa.v22i4.9

Dynamic Publishers, Inc., Acad. Publishers, Ltd. http://www.acadsol.eu/caa

\section{INTRODUCTION}

In this work, we study the following coupled reaction-diffusion system: 


$$
(I)\left\{\begin{aligned}
\frac{\partial u}{\partial t} & =\frac{\partial^{2} u}{\partial x^{2}}-u v^{n}, \quad x \in R^{1}, t>0 \\
\frac{\partial v}{\partial t} & =D \frac{\partial^{2} v}{\partial x^{2}}+u v^{n}
\end{aligned}\right.
$$

where $n>1$ and $D>0$. The system describes unstirred chemical reaction of the form

$$
A+n B \rightarrow(n+1) B
$$

called autocatalytic chemical reaction of order $n+1$, with $u$ the density of chemical species $A$, called reactant, and $v$ of of $B$, called autocatalyst. It is well documented in literature that this type of reactions appear in wide range of real-world chemical reactions such as almost isothermal combustion in carbon-sulphide-oxygen reaction, [17], IAA reaction [13] and hydroxylaminenitrate reaction [12]. Other applications include idealized Brusselator and clock reactions such as Belousov-Zhabotinsky and Bray-Liebhafsky reactions, $[7,8,18]$.

More importantly, in various models of biological pattern formation to simulate the experiments which for the first time realizing the Turing mechanism of pattern formation, whether it is CIMA or Gray-Scott, $A+2 B \rightarrow 3 B$ is a key step, see $[10,11]$.

The purpose of this work is to study traveling wave of (I) in the extreme cases of $0<D \ll 1$ and $D \gg 1$. For a traveling wave of $(\mathrm{I}), u(x, t)=$ $u(z), \quad v(x, t)=v(z)$, where $z=x-c t$, the resulting ODE system is:

$$
\left\{\begin{array}{l}
u^{\prime \prime}+c u^{\prime}-u v^{n}=0 \\
D v^{\prime \prime}+c v^{\prime}+u v^{n}=0
\end{array}\right.
$$

where $c>0$ is a constant. If we subscribe

$$
\lim _{z \rightarrow-\infty}(u, v)=(0, a), \quad a>0
$$

simple computation involving the addition of the two equations and integration on $(-\infty, z]$ yields

$$
u^{\prime}+D v^{\prime}+c(u+v-a)=0 .
$$


This, plus the property that $u$ is increasing before $v$ hits its first zero, gives that for a traveling wave solution,

$$
\lim _{z \rightarrow \infty}(u, v)=(a, 0) .
$$

The traveling wave problem, after replacing the 1st equation in (1.1) by (1.2), is the following 3rd order dynamical system:

$$
\begin{cases}u^{\prime}+D v^{\prime}=c(a-u-v), & \forall z \in \mathbb{R}, \\ D v^{\prime \prime}+c v^{\prime}=-u v^{n}, & \forall z \in \mathbb{R}, \\ \lim _{z \rightarrow-\infty}(u, v)=(0, a), & \lim _{z \rightarrow \infty}(u, v)=(a, 0) .\end{cases}
$$

It turns out the traveling wave problem of (I) is of the mono-stable type for scalar equation with a minimum positive speed $c_{\min }(D, a, n)$, that is a traveling wave solution exists iff $c \geq c_{\min }(D, a, n)$, see [5]. The main effort is then to prove sharp bound on minimum speed, because the minimum speed traveling wave is the most stable one.

In the general setting of $D$ staying away from zero and is bounded from above, satisfactory bounds are derived in [3] and [4]. In particular, it is shown that there exist $c_{1}(D, a, n)>0$ and $c_{2}(D, a, n)>0$ such that if $c \geq c_{2}(D, a, n)$ there exists a traveling wave of speed $c$, whereas if $c<c_{1}(D, a, n)$ there exists no traveling wave of spped $c$. But, the results there break down when $0<$ $D \ll 1$ or $D \gg 1$.

The purpose of the present work is to carefully reformulate the traveling wave problem for these extreme cases and derive explicit lower bounds of $c$ for existence, and upper bounds for $c$ for non-existence.

System (I) has a lot of interesting dynamics and the study of global dynamics of solutions with initial values decay sufficiently fast as well as the study of stability of traveling waves investigated in [2], [9], [15] and [16].

The plan of this paper is that we investigate the case of $0<D \ll 1$ in Section 2 and that of $D \gg 1$ in Section 3.

\section{THE CASE OF $0<D \ll 1$}

In this section we study the case of $0<D \ll 1$ with general boundary value

$$
\lim _{z \rightarrow-\infty}(u, v)=(0, a), \lim _{z \rightarrow-\infty}(u, v)=(a, 0),
$$


where $a>0$. Let

$$
u(z)=\bar{u}\left(a^{n / 2} z\right) a, \quad v(z)=\bar{v}\left(a^{n / 2} z\right) a,
$$

then

$$
\begin{array}{ll}
u^{\prime}(z)=\bar{u}^{\prime}\left(a^{n / 2} z\right) a^{n / 2+1}, & u^{\prime \prime}(z)=\bar{u}^{\prime \prime}\left(a^{n / 2} z\right) a^{n+1}, \\
v^{\prime}(z)=\bar{v}^{\prime}\left(a^{n / 2} z\right) a^{n / 2+1}, & v^{\prime \prime}(z)=\bar{v}^{\prime \prime}\left(a^{n / 2} z\right) a^{n+1} .
\end{array}
$$

Hence, after dropping " -", we have

$$
\begin{cases}u^{\prime}+D v^{\prime}=\frac{c}{a^{n / 2}}(1-u-v), & \forall z \in \mathbb{R}, \\ D v^{\prime \prime}+\frac{c}{a^{n / 2}} v^{\prime}=-u v^{n}, & \forall z \in \mathbb{R}, \\ \lim _{z \rightarrow-\infty}(u, v)=(0,1), & \lim _{z \rightarrow \infty}(u, v)=(1,0) .\end{cases}
$$

By Theorem 1.3 of [3], there exist $c_{1}(D, n)$ and $c_{2}(D, n)$ such that if

$$
\frac{c}{a^{n / 2}}<c_{1}(D, n),
$$

there exists no traveling wave of speed $c$; but if

$$
\frac{c}{a^{n / 2}} \geq c_{2}(D, n)
$$

there exists a traveling wave of speed $c$, and both limits

$$
\lim _{D \rightarrow 0} \frac{c_{1}(D, n)}{D}, \quad \lim _{D \rightarrow 0} \frac{c_{2}(D, n)}{D}
$$

are finite and positive, depending only on $n$. It is clear from the above that if $a$ varies with $D$, then

$$
\begin{array}{cccc}
c_{\min }(D, a, n) \rightarrow 0 \quad \text { if } & a^{n / 2} D \rightarrow 0 \quad \text { as } & D \rightarrow 0, \\
c_{\text {min }}(D, a, n) \rightarrow \infty & \text { if } \quad & a^{n / 2} D \rightarrow \infty \quad \text { as } & D \rightarrow 0 .
\end{array}
$$

Therefore, the interesting case is $a^{n / 2} D$ has a finite and positive limit as $D \rightarrow$ 0 , and without loss of generality, we assume $a^{n / 2} D=1$. Our purpose is to derive explicit bounds under the assumption $0<D \ll 1$. The system (2.1) now takes the form

$$
\begin{cases}D^{-1} u^{\prime}+v^{\prime}-c(1-u-v)=0, & \forall z \in \mathbb{R}, \\ v^{\prime \prime}+c v^{\prime}+D^{-1} u v^{n}=0, & \forall z \in \mathbb{R}, \\ \lim _{z \rightarrow-\infty}(u, v)=(0,1), & \lim _{z \rightarrow \infty}(u, v)=(1,0) .\end{cases}
$$


To facilitate further analysis, we follow the idea of [3] to make further change of variables, which will reduce (2.2) to a 2 nd order dynamical system. Let

$$
\begin{gathered}
y=c z, \quad \alpha(y)=1-v(z), \quad \beta(y)=\frac{1}{c^{2} D} u(z) . \\
\alpha^{\prime}(y)=-\frac{1}{c} v^{\prime}(z), \quad \alpha^{\prime \prime}(y)=-\frac{1}{c^{2}} v^{\prime \prime}(z) \quad \beta^{\prime}(y)=\frac{1}{c^{3} D} u^{\prime}(z) .
\end{gathered}
$$

We have

$$
\begin{cases}\alpha^{\prime \prime}+a^{\prime}(y)=\beta(1-\alpha)^{n} & \forall y \in \mathbb{R}, \\ \beta^{\prime}(y)=\frac{1}{c^{2}}\left(\alpha^{\prime}+\alpha\right)-D \beta & \forall y \in \mathbb{R} .\end{cases}
$$

Further, use $\alpha$ as the independent variable, $Q(\alpha)=\alpha_{y}(y), \quad B(\alpha)=\beta(y)$, we get

$$
(I I) \begin{cases}Q Q^{\prime}+Q=B(1-\alpha)^{n}, & \forall 0 \leq \alpha<1 \\ Q B^{\prime}=\frac{1}{c^{2}}(Q+\alpha)-D B, & 0 \leq \alpha<1 \\ B(0)=Q(0)=0, & B(\alpha)>0, \quad Q(\alpha)>0 \quad \text { on } \quad(0,1) .\end{cases}
$$

It is a system of 2nd order non-autonomous system ODEs.

For $D=1,(1.2)$ with $a=1$ can be further simplified to yield $u+v \equiv 1$. In this case, (2.2) is reduced to a single equation,

$$
\begin{cases}v^{\prime \prime}+c v^{\prime}+(1-v) v^{n}=0 & \forall z \in \mathbb{R}, \\ \lim _{z \rightarrow-\infty} v=1, & \lim _{z \rightarrow \infty} v=0 .\end{cases}
$$

Similarly, following the same procedure as above for general case of $D$, we have

$$
\begin{cases}P^{\prime} P+P=\frac{\alpha}{c^{2}}(1-\alpha)^{n} & \forall \alpha \in(0,1), \\ P(0)=0, \quad P(\alpha)>0 & \text { on }(0,1) .\end{cases}
$$

The classical result on the existence of traveling wave solution is summarized in the following proposition.

Proposition 2.1. There exists $c(n)>0$ such that (2.3) has a solution iff $c \geq c(n)$. In addition, $c(n)$ decreases with $n$ and $c(1)=2, \quad c(2)=1 / \sqrt{2}$. 
Now, we go back to (II).

Lemma 2.2. Suppose $D>0, c>0$. There exists a unique solution of (II). In addition,

$$
Q(\alpha)=\mu \alpha+O\left(\alpha^{2}\right), \quad B(\alpha)=\mu(1+\mu) \alpha+O\left(\alpha^{2}\right) \quad \text { as } \alpha \searrow 0,
$$

where $\mu=\left(\sqrt{4+c^{2} D^{2}}-c D\right) / 2 c$. Moreover, the following properties hold.

(i) $B^{\prime}(\alpha)>0 \quad \forall \alpha \in(0,1) \quad$ and

(ii) there exist a traveling wave solution iff $Q(1)=0$.

The proof of the lemma follows the same procedure as in Lemma 2.2 of [3], we omit the details.

Lemma 2.3. Suppose $0<D<1$. Then $B(\alpha)>\mu(1+\mu) \alpha$ and

$$
\mu \alpha(1-\alpha)^{n} \leq Q(\alpha) \leq \mu \alpha \quad \text { in }[0,1)
$$

Proof. First, we show $B \geq \mu(Q+\alpha)$ in [0,1]. Direct computation shows

$$
\begin{aligned}
Q[B-\mu(Q+\alpha)]^{\prime} & =\frac{1}{c^{2}}(Q+\alpha)-\mu B(1-\alpha)^{n}-D B \\
& =-\left[D+\mu(1-\alpha)^{n}\right][B-\mu(Q+\alpha)+(Q+\alpha)] \\
& +\left(\frac{1}{c^{2}}-\mu^{2}(1-\alpha)^{n}-\mu D\right) \\
& >\left[D+\mu(1-\alpha)^{n}\right][B-\mu(Q+\alpha)+(Q+\alpha)] \quad \text { in }(0,1),
\end{aligned}
$$

since $1 / c^{2}=\mu^{2}+\mu D$. For $0<\alpha \ll 1$, Taylor expansion at $\alpha=0$ shows

$$
\begin{aligned}
& B(\alpha)=\mu(1+\mu) \alpha+\frac{\mu^{2}(1+\mu)(1-D) n}{\mu(1-D)+(2 \mu+D)(1+3 \mu)} \alpha^{2}+o\left(\alpha^{2}\right), \\
& Q(\alpha)=\mu \alpha-\frac{2 \mu+D}{\mu(1-D)} \frac{\mu^{2}(1+\mu)(1-D) n}{\mu(1-D)+(2 \mu+D)(1+3 \mu)}+o\left(\alpha^{2}\right) .
\end{aligned}
$$

Hence, $B>\mu(Q+\alpha)$ for $0<\alpha \ll 1$. An application of Gronwall's inequality using (2.4) yields

$$
B(\alpha)>\mu(1+\mu) \alpha \quad \text { on }(0,1) .
$$

Next, it is easy to prove that

$$
B(\alpha)(1-\alpha)^{n / 2} \leq \mu(Q(\alpha)+\alpha) \quad \text { on }(0,1) .
$$


This follows from the same argument as in Lemma 2.5 in [4], we refer the reader to that article. The two inequalities on $Q$ then can be proved by the same procedure of computing

$$
\left[Q-\eta \alpha(1-\alpha)^{\delta}\right]^{\prime}=-1+\frac{B(1-\alpha)^{n}}{Q}-\eta(1-\alpha)^{\delta}+\eta \delta \alpha(1-\alpha)^{\delta-1}
$$

$Q(\alpha) \leq \mu \alpha$ is proved by the use of (2.6) by taking $\eta=\mu$ and $\delta=0 ; Q(\alpha) \geq$ $\mu \alpha(1-\alpha)^{n}$ by the use of (2.5) and by taking $\eta=\mu$ and $\delta=n$, respectively. Finally,

$$
\begin{aligned}
Q[B(\alpha)-\mu(1+\mu) \alpha]^{\prime} & =\frac{1}{c^{2}}(Q+\alpha)-D B-\mu(1+\mu) Q \\
& =-D[B(\alpha)-\mu(1+\mu) \alpha]+[\mu(1+\mu)-D \mu(1+\mu)] \alpha \\
& +[\mu(D+\mu)-\mu(1+\mu)] Q \\
& =-D[B(\alpha)-\mu(1+\mu) \alpha]+\mu(D-1)(Q-\mu \alpha) \\
& \geq-D[B(\alpha)-\mu(1+\mu) \alpha],
\end{aligned}
$$

since $1 / c^{2}=\mu(\mu+D)$. Another use of Gronwall's inequality yields the desired result.

Lemma 2.4. Suppose $0<D<1$ and $1<n<2$. Then for any

$$
\begin{gathered}
\lambda>\max \left(\mu, \frac{1}{c^{2}[(n-1) \mu+D n]}\right), \\
B(1-\alpha)^{n-1} \leq \lambda(Q+\alpha) \quad \text { on }[0,1] .
\end{gathered}
$$

Proof. Elementary computation shows

$$
\begin{aligned}
Q\left[(1-\alpha)^{n-1} B-\lambda(Q+\alpha)\right]^{\prime} & =(1-\alpha)^{n-1}\left(\frac{1}{c^{2}}(Q+\alpha)-D B\right) \\
& -(n-1)(1-\alpha)^{n-2} Q B-\lambda B(1-\alpha)^{n} \\
\leq & -\left[D+\mu(1-\alpha)+(n-1) \mu \alpha(1-\alpha)^{n-1}\right] \\
& \times\left[(1-\alpha)^{n-1} B-\lambda(Q+\alpha)\right] \\
& +(Q+\alpha) H(\alpha),
\end{aligned}
$$

since $Q>\mu \alpha(1-\alpha)^{n}$ on $(0,1)$. Here

$$
H(\alpha)=\frac{1}{c^{2}}-\lambda^{2}(1-\alpha)^{2-n}-\lambda \mu(n-1) \alpha-\lambda D(1-\alpha)^{1-n} .
$$


The lemma holds if $H(\alpha) \leq 0$ on $[0,1)$. It is clear that $H(0)<0$ if $\lambda>\mu$. In addition,

$$
\begin{gathered}
H(\alpha) \leq \frac{1}{c^{2}}-\lambda^{2}(1-\alpha)-\lambda \mu(n-1) \alpha-\lambda D(1-\alpha)^{1-n} \equiv g(\alpha) . \\
g^{\prime}(\alpha)=\lambda^{2}-\lambda \mu(n-1)-\lambda D(n-1)(1-\alpha)^{-n} .
\end{gathered}
$$

If $\lambda \leq(\mu+D)(n-1)$, then $g(\alpha) \leq 0$ on $(0,1)$ and we are done. Otherwise, since $g^{\prime \prime}(\alpha)<0$ on $(0,1)$, there exists an $\alpha_{0} \in(0,1)$ which is a maximum point. At this point,

$$
\begin{aligned}
& \lambda^{2}=\lambda \mu(n-1)+\lambda D(n-1)(1-\alpha)^{-n}, \\
g(\alpha)= & \frac{1}{c^{2}}-\lambda \mu(n-1)(1-\alpha)-\lambda D(n-1)(1-\alpha)^{1-n} \\
& -\lambda \mu(n-1) \alpha-\lambda D(1-\alpha)^{1-n} \\
= & \frac{1}{c^{2}}-\lambda \mu(n-1)-\lambda D n(1-\alpha)^{1-n}<0,
\end{aligned}
$$

if $\lambda$ satisfies (2.7). This proves the lemma.

Theorem 2.5. Suppose $0<D \ll 1$.

(i) If $n \geq 2$, there exists a traveling wave solution to (II) with speed $c$ if $c>4$.

(ii) If $1<n<2$, there exists a traveling wave solution to (II) with speed $c$ if $c>4 /(n-1)$.

(iii) There exists no traveling wave solution to (II) with speed $c$ if

$$
c<\frac{c(n) \sqrt{2}}{\sqrt{c^{2}(n)-c(n) \sqrt{c^{2}(n)+4}+2}} .
$$

Proof. We shall proof existence first. Suppose $n \geq 2$. It is easy to verify that

$$
I(\alpha)=[Q-\eta \alpha(1-\alpha)]^{\prime}=-1+\frac{B}{(1-\alpha)^{n}} Q-\eta(1-\alpha)+\eta \alpha .
$$

If $\eta>\mu, Q-\eta \alpha(1-\alpha)<0$ for $0<\alpha \ll 1$. Furthermore, when $Q=\eta \alpha(1-\alpha)$, $0<\alpha<1$, by $(2.6)$,

$$
I(\alpha) \leq-1+\frac{\mu(Q+\alpha)(1-\alpha)^{n / 2}}{\eta \alpha(1-\alpha)}-\eta(1-2 \alpha)
$$




$$
\begin{aligned}
& =-1+\frac{\mu}{\eta}(1-\alpha)^{n / 2-1}+\mu(1-\alpha)^{n / 2}-\eta(1-2 \alpha) \\
& \leq-1+\frac{\mu}{\eta}+\mu(1-\alpha)-\eta(1-2 \alpha) \equiv g(\alpha) .
\end{aligned}
$$

Apparently, if $\mu \leq 1 / 4$, there exists $\eta>\mu$ such that $g(\alpha)<0$ in $[0,1]$. Hence, $Q<\eta \alpha(1-\alpha)$ on $(0,1)$ if $\mu \leq 1 / 4$.

If $C>4$ it is easy to check that for all $0<D \ll 1, \mu<1 / 4$, and hence $Q(1)=0$. That is, we have a traveling solution of speed $c$.

Next, $1<n<2$. In this case, by Lemma 2.4,

$$
B(1-\alpha)^{n} \leq \lambda(Q+\alpha)(1-\alpha)
$$

if $\lambda$ satisfies (2.7). By repeating the above procedure we can show that $Q<$ $\eta \alpha(1-\alpha)$ on $(0,1)$ if $\lambda \leq 1 / 4$.

The non-existence part is a direct consequence of using (2.5) in the 1 st equation of (II) and in comparison with the single equation case.

\section{THE CASE OF $D \gg 1$}

In this section we study the case of $D \gg 1$ with general boundary value

$$
\lim _{z \rightarrow-\infty}(u, v)=(0, a), \lim _{z \rightarrow-\infty}(u, v)=(a, 0),
$$

where $a>0$. Let

$$
u(z)=\bar{u}\left(a^{n / 2} z\right) a, \quad v(z)=\bar{v}\left(a^{n / 2} z\right) a
$$

then

$$
\begin{array}{ll}
u^{\prime}(z)=\bar{u}^{\prime}\left(a^{n / 2} z\right) a^{n / 2+1}, & u^{\prime \prime}(z)=\bar{u}^{\prime \prime}\left(a^{n / 2} z\right) a^{n+1} \\
v^{\prime}(z)=\bar{v}^{\prime}\left(a^{n / 2} z\right) a^{n / 2+1}, & v^{\prime \prime}(z)=\bar{v}^{\prime \prime}\left(a^{n / 2} z\right) a^{n+1} .
\end{array}
$$

Hence, after dropping " -", we have

$$
\begin{cases}u^{\prime}+D v^{\prime}=\frac{c}{a^{n / 2}}(1-u-v), & \forall z \in \mathbb{R}, \\ D v^{\prime \prime}+\frac{c}{a^{n / 2}} v^{\prime}=-u v^{n}, & \forall z \in \mathbb{R} \\ \lim _{z \rightarrow-\infty}(u, v)=(0,1), & \lim _{z \rightarrow \infty}(u, v)=(1,0) .\end{cases}
$$


By Theorem 1.4 of [3], there exist $c_{3}(D, n)>0$ and $c_{4}(D, n)>0$ such that if

$$
\frac{c}{a^{n / 2}}<c_{3}(D, n)
$$

there exists no traveling wave of speed $c$; but if

$$
\frac{c}{a^{n / 2}} \geq c_{4}(D, n)
$$

there exists a traveling wave of speed $c$, and the limits

$$
\lim _{D \rightarrow 0} \frac{c_{3}(D, n)}{\sqrt{D}}, \quad \lim _{D \rightarrow \infty} \frac{c_{4}(D, n)}{\sqrt{D}}
$$

are both finite and positive, depending only on $n$. It is clear from the above that if $a$ varies with $D$, then

$$
\begin{aligned}
& c_{\min }(D, a, n) \rightarrow 0 \quad \text { if } \quad a^{n} D \rightarrow 0 \quad \text { as } \quad D \rightarrow \infty, \\
& c_{\min }(D, a, n) \rightarrow \infty \quad \text { if } \quad a^{n} D \rightarrow \infty \quad \text { as } \quad D \rightarrow \infty \text {. }
\end{aligned}
$$

Therefore, the interesting case is $a^{n} D$ has a finite and positive limit as $D \rightarrow \infty$, and without loss of generality, we assume $a^{n} D=1$. Our purpose is to derive explicit bounds under the assumption $D \gg 1$. The system (3.1) now takes the form

$$
\begin{cases}D^{-1} u^{\prime}+v^{\prime}-c D^{1 / 2}(1-u-v)=0, & \forall z \in \mathbb{R}, \\ D v^{\prime \prime}+c D^{1 / 2} v^{\prime}+u v^{n}=0, & \forall z \in \mathbb{R}, \\ \lim _{z \rightarrow-\infty}(u, v)=(0,1), & \lim _{z \rightarrow \infty}(u, v)=(1,0) .\end{cases}
$$

Let

$$
\begin{gathered}
y=c D^{-1 / 2} z, \quad \alpha(y)=1-v(z), \quad \beta(y)=\frac{1}{c^{2} D} u(z) . \\
-\alpha^{\prime}(y) c D^{-1 / 2}=v^{\prime}(z), \quad \alpha^{\prime \prime}(y) c^{2} D^{-1}=-v^{\prime \prime}(z) \quad \beta^{\prime}(y) c^{3} D^{1 / 2}=u^{\prime}(z) .
\end{gathered}
$$

We have

$$
\begin{cases}\alpha^{\prime \prime}+a^{\prime}(y)=\beta D(1-\alpha)^{n} & \forall y \in \mathbb{R}, \\ \beta^{\prime}(y)=\frac{1}{c^{2}}\left(\alpha^{\prime}+\alpha\right)-D \beta & \forall y \in \mathbb{R} .\end{cases}
$$


Further, use $\alpha$ as the independent variable, $Q(\alpha)=\alpha_{y}(y), \quad B(\alpha)=\beta(y)$, we arrive at exactly

$$
(I I I) \begin{cases}Q Q^{\prime}+Q=D B(1-\alpha)^{n} & \forall 0 \leq \alpha<1, \\ Q B^{\prime}=\frac{1}{c^{2}}(Q+\alpha)-D B, & 0 \leq \alpha<1, \\ B(0)=Q(0)=0, & B(\alpha)>0, \quad Q(\alpha)>0 \quad \text { on } \quad(0,1) .\end{cases}
$$

It is again a system of 2nd order non-autonomous system ODEs.

Our main result in this case is the following theorem.

Theorem 3.1. Suppose $D \gg 1$ and $n \geq 1$.

(i) If $c<c(n)$, there exists no traveling wave to (III). But, if

(ii) c satisfies

$$
c>\frac{\sqrt{c(n)}}{\sqrt{2}}\left(\sqrt{c^{2}(n)+4}+c(n)\right)^{1 / 2},
$$

there exists a traveling wave to (III).

The main technical result in this case is the following lemma.

Lemma 3.2. Suppose $D>1$ and $n>1$. Then, there exists a unique solution of (III) with

$$
Q(\alpha)=\nu \alpha+O\left(\alpha^{2}\right), \quad B(\alpha)=\frac{\nu(1+\nu)}{D}+O\left(\alpha^{2}\right) \alpha \quad \text { as } \alpha \searrow 0 .
$$

Moreover,

(i) $D B(\alpha) \geq \alpha / c^{2}$, consequently, there is no traveling wave solution when $c<c(n)$,

(ii) $D B(\alpha)<\nu(1+\nu) \alpha, \quad Q(\alpha)<\nu \alpha$ on $(0,1)$.

Since the proof of Lemma 3.2 is in the same line of argument as Lemma 3.1 and Lemma 3.2 in [3], we omit the details here.

Proof of Theorem 3.1. It is a direct consequence of Lemma 3.2 and elementary computation. 


\section{ACKNOWLEDGMENTS}

The author wishes to thank Prof. Gaofeng Zheng for stimulating discussion.

\section{REFERENCES}

[1] P. Bates, P. Fife, X. Ren and X. Wang, Traveling waves in a convolution model for phase transitions, Arch. Rational Mech. Anal., 138 (1997), 105-136.

[2] J. Bricmont, A. Kupiainen and J. Xin, Global large time self-similarity of a thermal-diffusive combustion system with critical nonlinearity, $J$. Differential Equations, 130 (1996), 9-35.

[3] Xinfu Chen, and Yuanwei Qi, Sharp estimates on minimum traveling wave speed of reaction diffusion systems modeling autocatalysis. SIAM J. Math. Anal., 39 (2007), 437-448.

[4] X. F. Chen and Yuanwei Qi. Travelling waves of auto-catalytic chemical reaction of general order-an elliptic approach, J. Differential Equations, 246 (2009), 3038-3057.

[5] X. F. Chen, G. R. Liu and Yuanwei Qi. The existence of minimum speed of traveling wave solutions to a Non-KPP isothermal diffusion system, to appear in J. Differential Equations, (2017).

[6] X. F. Chen, Yuanwei Qi and Y. J. Zhang, Existence of traveling waves of Auto-Catalytic systems with decay, J. Differential Equations, 260 (2016), 7982-7999.

[7] P. Gray and S. K. Scott, Autocatalytic reactions in the isothermal, continuous stirred tank reactor: Oscillations and instabilities in the system $A+2 B \rightarrow 3 B, B \rightarrow C$, Chem. Eng. Sci., 39 (1984), 1087-1097.

[8] P. Gray, Instabilities and oscillations in chemical reactions in closed and open systems, Proc. Roy. Soc. A, 415 (1988), 1-34. 
[9] Y. Li and Y.P. Wu, Stability of Traveling Front Solutions with Algebraic Spatial Decay for Some Autocatalytic Chemical Reaction Systems, SIAM J. Math. Anal, 44 (2012), 1474-1521.

[10] K.-J. Lin, W.D. McCormick, J.E. Pearson, and H.L. Swinney, Experimental observation of self-replicating spots in a reaction-diffusion system, Nature, 369 (1994), 215-218.

[11] J. E. Pearson, Complex patterns in a simple system, Science, 261 (1993), 189-192.

[12] R. J. Gowland and G. Stedman, A novel moving boundary reaction involving hydoxylamine and nitric acid, J. Chem. Soc. Chem. Comm., 10(1983), 1038-1039.

[13] A. Saul and K. Showalter, Propagating reaction-diffusion fronts, in Oscillations and traveling waves in chemical systems, (Ed R. J. Field and M Burgern) Wiley, New York, 1984.

[14] V. G. E. E. Sel'kov , Euro. J. Biochem. 4 (1968), 79.

[15] Junping Shi and Xuefeng Wang, Hair-triggered instability of radial steady states, spread and extinction in semilinear heat equations, $J$. Differential Equations, 231 (2006), 235-251.

[16] J. Xin, Front propagation in heterogeneous media, SIAM Rev., 42 (2000), 161-230.

[17] V. G. Voronkov and N. N. Semenov, Zh. Fiz. Khim. 13 (1939), 1695.

[18] A. N. Zaikin and A. M. Zhabotinskii, Concentration wave propagation in two-dimensional liquid-phase self-organising systems, Nature, 225 (1970), 535-537. 
